\title{
Using email questionnaires for research: Good practice in tackling non-response
}

Received: 14th March, 2006

\section{Nina Michaelidou}

is Lecturer in Marketing at Birmingham Business School, University of Birmingham. She holds a PhD from Warwick in consumer behaviour and has written papers for European and US journals on variety seeking, involvement and channel characteristics. Dr Michaelidou teaches marketing and consumer behaviour on a range of degree programmes.

\section{Sally Dibb}

is Professor of Marketing at the Open University Business School, Milton Keynes, UK. She has authored five books, including the best-selling textbook 'Marketing: Concepts and Strategies', now in its fifth edition. Her research is focused on market segmentation and marketing planning areas in which she has published widely.

\begin{abstract}
The use of email as an alternative to mail for collecting data provides new opportunities for researchers worldwide. While researchers have extensively focused on identifying the advantages and disadvantages of online surveys, empirical evidence on response rates to email questionnaires remains scarce. A number of factors have been cited to explain this, including lack of anonymity and incentives. As response rates tend to vary in different contexts, additional empirical evidence is needed to demonstrate how improvements in such rates can be achieved in the context of specific research. This will assist researchers in designing email surveys that meet the designated research objectives and achieve adequate response. This paper describes the use of an email questionnaire to collect data for a consumer study. It explores how non-response can be tackled by selecting an appropriate sampling frame, through the choice of questionnaire type, by using incentives and by ensuring anonymity. The paper concludes by offering a five-point good practice checklist for researchers involved in designing online surveys.
\end{abstract}

Nina Michaelidou

University of Birmingham

School

University House,

Edgbaston, Birmingham

UK

Email:

n.michaelidou@bham.ac.uk

\section{INTRODUCTION}

The selection of a survey medium for marketing research often raises issues of cost and handling. The cheapest method in terms of per-contact cost is generally considered to be the mail questionnaire, as this approach does not involve field staff, as in the cases of telephone surveys or personal interviews. ${ }^{1}$ However, mailing involves physical effort associated with collating questionnaires and stuffing and addressing envelopes. This may be time-consuming and costly, particularly where the sample size is large. To avoid such costs, the use of email as a survey medium seems particularly attractive. ${ }^{2}$

The use of email as an alternative to mail for data collection provides new opportunities for researchers worldwide. Within this context, research has been undertaken to examine the usefulness and efficiency of email as a survey medium, 
comparing it with telephone and postal surveys. ${ }^{3}$ There is general agreement about the wide-ranging benefits of email surveys, which include low transmission and collection costs, immediate transmission and response and ease of use. ${ }^{4-10}$ Despite these advantages, email surveys have several drawbacks.

According to Ranchhod and Zhou, ${ }^{11}$ a major concern is the low response rates associated with the method. Dommeyer and Moriarty ${ }^{12}$ and Mehta and Sivadas ${ }^{13}$ compared email surveys with traditional mail surveys and reported disappointing response rates, which they attributed to a lack of anonymity, low use of incentives and poor design of email questionnaires. Although some authors argue that response rates depend on the study context, ${ }^{14-16}$ the general consensus is that email questionnaires tend to have poor return rates compared with postal surveys. ${ }^{17,18}$

Examining the effectiveness of email surveys by identifying the inherent advantages and disadvantages may assist researchers seeking to use this method of data collection. It does not, however, tackle the problem of non-response. Current research on factors affecting non-response focuses on permission marketing, ${ }^{19}$ pre-notifications, ${ }^{20}$ and personalisation. ${ }^{21,22}$ The personalisation of salutations in email surveys has been found to be particularly effective in increasing response rates, with improvements of 7.8 per cent recorded. ${ }^{23}$ According to Heerwegh, ${ }^{24}$ personalisation affects the level of perceived anonymity and leads to a higher self-commitment, thus reducing the break-off rate.

However, as personalised invitations to online surveys cannot ensure anonymity, there is a danger that individuals consider them to be intrusive or unsolicited.

Despite extant research, empirical evidence on factors affecting response rates and response quality for online surveys remains limited. This has the potential to discourage the use of email and web-based data collection tools, even though their time and cost advantages are known to impact upon efficiency and effectiveness. ${ }^{25}$ The provision of additional empirical evidence on response rates for email questionnaires may make it easier for researchers to select a survey medium. This is important, as response is closely related to a study's sampling frame, to the type of email questionnaire, and to the use of incentives, ${ }^{26}$ all of which are likely to vary across studies.

This paper focuses on the consumer context, using an application of an email survey and a literature review as the basis for developing a series of guidelines for researchers seeking to use this method of data collection. The paper has three aims. First, to contribute to the accumulation of empirical knowledge on the usefulness of email questionnaires in meeting particular research objectives. Secondly, to suggest ways of tackling non-response to email surveys by (1) targeting the appropriate sample, (2) selecting particular type of email questionnaire, and (3) by using incentives. Thirdly, to provide researchers wishing to adopt this method with specific guidelines or recommendations for best practice in designing email questionnaires.

\section{THE STUDY}

\section{Sampling frame}

The subject of the study was clothing purchase. The questions measured consumer attitudes and behaviour towards shopping of clothes via three different channels (retail stores, catalogues, the internet). The research objectives shaped the sampling frame, which required potential respondents to have experience of shopping for clothes 
via multiple channels. Because of the uncertainty surrounding the quality of mailing lists and the consequent problems of potential sampling bias, it was considered advantageous to have a company's customer database as the sampling frame. The sample was derived from the large customer database of NEXT plc, one of the largest and best-known UK clothing retailers. The NEXT brand is available to purchase from retail stores, printed catalogues, the internet and digital television. NEXT's database consists of more than 100,000 individuals who have registered their personal information (including email addresses) with the company. Some of these individuals already have an online and/or catalogue shopping record with NEXT plc and some do not. However, all were assumed to shop from the high street retail stores. The retailer only agreed to cooperate on the basis that direct access to its customer database would not be allowed by third parties. Instead, the company used agreed principles to derive the sample randomly from their database and to make contact with targeted respondents.

Although the use of this type of non-probability sample may raise concerns about generalisability, it can be theoretically justified because it serves the research objectives. ${ }^{27}$ The cost benefits, convenience and speed of response outweigh any compromise in terms of sample coverage. Although the use of email is known to limit distribution to those individuals with access to computers and email accounts, this was not a disadvantage in this case as the profile of such respondents matched the sample requirements. Therefore, this type of sample was representative of the specific population in this particular research context. In other words, a central register of the research population existed and all members of the population had the chance to respond via email and web or by mail. Consequently, there were no concerns about external validity. Furthermore, a comparison of late and early respondents ruled out the possibility of non-response bias $^{28}$ on the basis of response to a number of variables.

Using a customer database as a sampling frame avoids the problems of outdated or inactive email addresses. It also ensures the quality of information about potential respondents who have the necessary computing skills and equipment to participate in a survey and who frequently access their email accounts. Individuals generally regard unsolicited survey emails from unknown sources as unacceptable. ${ }^{29}$ However, survey emails received from known sources, particularly those with which individuals are already registered, are less inhibiting and thus have a better chance of response. This is in line with previous research which suggests that consumers are less concerned about privacy in situations that involve familiar online vendors with whom they have established a relationship. ${ }^{30}$

\section{Type of email questionnaires for data collection}

Email questionnaires were selected due to their cost effectiveness, fast transmission and response turnaround..$^{31,32}$ Considerable thought was given to the type of email questionnaire. A key factor was the large number of questions involved in the study which affected the length of the questionnaire. Previous research on both online and mail surveys emphasises the relationship between length and response rate/quality. ${ }^{33-35}$ It is known that shorter questionnaires tend to attract more responses than longer questionnaires. To overcome potential non-response for the study, two 
alternative forms of email questionnaire were considered: an email message carrying questions as an attachment and an email with a URL embedded in the message inviting potential respondents to click on the link and complete the questionnaire.

The second option was chosen because the process of handling, completing and transmitting the questionnaire was simpler. Email messages carrying questionnaires as attachments require multiple steps on the part of the respondent and may discourage response. ${ }^{36}$ A URL-embedded questionnaire, on the other hand, seems less intrusive in that the potential respondent has the option of whether or not to visit the webpage to complete the questionnaire. Such an approach allows flexibility in font and layout, which encourages potential respondents. ${ }^{37}$ This is pertinent to both email and mail questionnaires. Although mixed results have previously been reported, it seems that questionnaire design may affect both response rates and response quality. ${ }^{38-40}$ However, a questionnaire's appearance may be greatly affected by the medium through which it is transmitted. In the case of email questionnaires, there are dangers in the research instrument appearing lengthy, plain or even unfriendly. ${ }^{41,42}$

The questionnaire was designed using a Microsoft Word-friendly program (Adobe Page Mill) which facilitated the completion process. The final research instrument did not take more than 15 minutes to complete. It was presented as a one-page document, through which respondents were able to scroll up and down. This minimised the waiting time required to 'turn' to the next page and made the questionnaire convenient to handle, easy and quick to complete. If presented in multiple pages, the questionnaire would have appeared longer. Consequently, potential respondents may have been discouraged. The layout of the questionnaire was carefully designed. Spaces were allowed between sections and questions. Closed-ended questions were widely used, employing three different types of response tags, including radio buttons, check boxes and text boxes. The colour and font of the questionnaire were chosen to appeal to potential respondents and to make the questionnaire appear friendly. Design features such as colour and images can facilitate the completion task and lead to efficiency and increased data quality. ${ }^{43}$ The questionnaire included an introduction which informed the respondents about the study as well as giving instructions and notes for completion. Once published as a webpage, rigorous testing was undertaken to ensure that responses could be smoothly transmitted to the sender.

Potential respondents received a URL address in the form of a hypertext link included in an email message written by the researcher and forwarded by the retailer. The email explained the purpose of the study and invited recipients to click on the hypertext link, which then invoked the web browser, presenting the individual with the web-based questionnaire posted on an academic website. Once completed, questionnaires were transmitted anonymously to the sender. Responses were received by email in a format which enabled their transfer to Microsoft Excel and then SPSS.

A pilot test to a sample of 300 individuals which preceded the main questionnaire launch was used to assess response rate potential and determine any problems. The pilot indicated a response rate of 20 per cent. However, some complaints were received by the retailer from customers who initially thought that their email addresses had been 
disclosed to third parties. To avoid a similar effect in the main questionnaire launch, the retailer agreed to send a direct message to potential respondents inviting them to participate in the study. The questionnaire was launched on a weekday morning and three weeks were allocated for their return. An

overwhelming 41.3 per cent of received responses arrived on the day of transmission. This is in line with Dibb et al. ${ }^{44}$ who found an equally high 'same day response' of 44 per cent, although Schillewaert et al. ${ }^{45}$ report a much lower figure of 15 per cent. High 'same day responses' may suggest that respondents decide fairly quickly whether or not they want to participate in the email survey. ${ }^{46}$ A steady and consistent rate of response was then received for the remainder of the first week. By the end of this period, 90 per cent of the final responses had been received. Overall, the response climbed to 20 per cent. Although this was adequate to meet the statistical objectives of the research, it is generally lower than that reported in other email surveys. However, it should be noted that the type of email questionnaire used (an email with URL embedded) and the nature of the particular sampling frame, mean that the response rates are not directly comparable with studies using different recruitment methods (eg newsgroups posting, webpage hyperlinks) as well as different samples ${ }^{47}$ (eg students, academic etc). This is in line with Schillewaert et al. ${ }^{48}$ who found that different recruitment methods tend to attract different response rates and respondents profiles.

Of all of the submitted questionnaires, 93 per cent were fully completed with no mistakes, indicating high response quality. Completion mistakes were generally avoided because the program used to design the questionnaire allowed greater control over the questionnaire layout. In addition, the type of tags employed enabled respondents to answer quickly and accurately, despite the length of the questionnaire.

\section{Topic of study, incentive, and anonymity}

The topic of study may also affect response to email questionnaires. Marketing researchers should ideally select a sampling frame of potential respondents that is likely to be interested in the topic being studied. This issue has not been systematically addressed in the literature. However, the limited research that does exist suggests that effective targeting by aiming the email questionnaires at potential respondents who are interested in the subject of study may increase response rates. ${ }^{49}$ Here, the expectation was that potential respondents would be interested in the email survey because of the positive steps they had taken to be included in the NEXT database. Indeed, the fact that adequate response rates were achieved would tend to support that effective targeting may be a way to tackle low response rates.

An incentive in the form of entry into a competition for $£, 100$ worth of vouchers was offered by the retailer to encourage response. Given the length of the questionnaire (100-items) the reward was considered essential. Lack of tangible incentives is generally considered as an inhibitor to email survey response. ${ }^{50,51}$ This is consistent with literature on mail surveys where incentives have been found to improve response rates. ${ }^{52}$

Incentives for email questionnaire completion can be used in the form of competitions for vouchers which are subsequently sent to the winner(s) in electronic form or via mail. In order to take advantage of incentives, respondents may be required to reveal their identity. In the case of this URL-embedded 
questionnaire, respondents could opt in for the voucher competition by submitting their email address at the end of the questionnaire. All respondents to the study submitted their email addresses to enter the prize draw. It seems reasonable to suppose that these respondents may have been less inhibited in providing these details because they were sending them to a company with which they were already registered. Respondents' email addresses were then gathered by the retailer who conducted a prize draw and informed the lucky winner.

Another factor frequently blamed for inhibiting email survey response ${ }^{53}$ is lack of anonymity. ${ }^{54}$ Most email surveys disclose respondents' email address and personal information causing privacy infringement. This is particularly the case where the email survey is unsolicited. ${ }^{55}$ This may cause frustration on the part of the respondents and complaints may be received from customers. Personalisation also decreases the level of perceived anonymity and perceived privacy. ${ }^{56}$ While personalised salutation messages have been found to increase access to web surveys and response rates, the extent to which such messages infringe anonymity has yet to be addressed. ${ }^{57}$ Even with personalised salutation messages, individuals may perceive email messages as highly intrusive if they have no relationship with the online vendor. $^{58,59}$

It is interesting to note that while the data collection described here was ongoing, NEXT received complaints from customers who thought that their email addresses had been disclosed to third parties. Indeed, personalisation was not adopted in this study as ensuring anonymity was considered an important parameter in encouraging response rates following complaints received in the piloting stage. This highlights the sensitivity of this matter and the need to minimise unsolicited email and internet surveys. However, the email invitation as well as the questionnaire design allowed responses to be received anonymously via email, except in the case where respondents wished to participate in the competition.

\section{GUIDELINES FOR BEST PRACTICE IN DESIGNING AN EMAIL SURVEY}

As a result of the study and literature described above, it is possible to develop a series of guidelines for researchers considering data collection using email questions. These recommendations are presented in the form of a five-point good practice checklist.

- Using email questionnaires with a URL embedded design enables simpler handling, completion and transmission. Potential respondents are able to access the research instrument by clicking on the hypertext link provided in the email invitation. Such questionnaires also facilitate the transmission of the data to the researcher in an email format.

- Ensuring anonymity and privacy can dramatically affect response rates. Email questionnaire design must adhere to the principle of anonymity and use mechanisms that enable this to be achieved.

- Individuals are more likely to respond to surveys that are relevant or of interest to them. Careful targeting of the email questionnaire to a sampling frame that exhibits interest in the topic of study is likely to increase response rates. The selection of the sampling frame must closely reflect the research objectives and subject under study.

- There may be advantages to deriving a sampling frame from a company's 
database if the profile matches the sample requirements. Such practice can increase response rates and turnaround time. There is also evidence that respondents are encouraged to reply to survey emails received from known sources, particularly those with which the individuals are already registered.

- Incentives should be used to encourage response rates, especially if the email questionnaires are lengthy. Potential respondents are likely to trade off their anonymity if incentives are used. The researcher can easily negotiate completion incentives if the sampling frame derives from a company's database.

\section{CONCLUSION}

Response rates to email questionnaires vary according to the study context. Various factors have been found to inhibit response to email or internet data collection. These factors include poor design of email questionnaires, lack of anonymity and completion incentives. By addressing these factors in the context of specific research objectives it may provide a way to tackle non-response to email questionnaires. Equally important in ensuring response to email surveys is the sampling frame of the research and the type of email questionnaire used. In the study described in this paper, an email questionnaire with URL-embedded design was used to target a sampling frame derived from a company's database. This type of questionnaire allowed flexibility in designing, transmitting, completing and finally handling the questionnaire. It also enabled anonymity of respondents, a factor often identified as an inhibitor of response rates in email and internet questionnaires. The questionnaire targeted a sampling frame which matched the sample requirements. This encouraged response rates and a fast turnaround, despite the length of the questionnaire which was also dealt with by offering a completion incentive. These findings have resulted in the development of a five-point good practice checklist for researchers considering the use of email questionnaires.

\section{References}

1 Tse, A. (1998) 'Comparing the response rate, response speed and response quality of two methods of sending questionnaires: e-mail versus mail', Journal of the Market Research Society, Vol. 40, No. 4, pp. 353-361.

2 Dibb, S., Rushmer, A. and Stern, P. (2001) 'New survey medium: Collecting marketing data with e-mail and the World Wide Web', Journal of Targeting, Measurement and Analysis for Marketing, Vol. 10, No. 1, pp. 17-25.

3 Coderre, F., Mathieu, A. and St-Laurent, N. (2004) 'Comparison of the quality of quantitative data obtained through telephone, postal and e-mail surveys', International Journal of Market Research, Vol. 46, No. 3, pp. 347-357.

4 Ilieva, J., Bacon, S. and Healeay, N. M. (2002) 'Online surveys in marketing research: Pros and cons', International Journal of Market Research, Vol. 44, No. 4, pp. 440-453.

5 Dibb et al., ref. 2 above.

6 Nebenzahl, I. D. and Jaffe, E. D. (1995) 'Facsimile transmission versus mail delivery of self-administered questionnaires in industrial surveys', Industrial Marketing Management, Vol. 24, No. 3, pp. 167-175.

7 Tse, ref. 1 above.

8 Wilson, A. and Laskey, N. (2003) 'Internet based marketing research: A serious alternative to traditional research methods?', Marketing Intelligence and Planning, Vol. 21, No. 2, pp. 79-84.

9 McDonald, H. and Adam, S. (2003) 'A comparison of online and postal data collection methods in marketing research', Marketing Intelligence and Planning, Vol. 21, No. 2, pp. 85-95.

10 Roy, A. and Berger, P. D. (2005) 'E-mail and mixed mode database surveys revisited: Exploratory analyses of factors affecting response rates', Database Marketing and Customer Strategy Management, Vol. 12, No. 2, pp. 153-171.

11 Ranchhod, A. and Zhou, F. (2001) 'Comparing respondents of e-mail and mail surveys: Understanding the implications of technology', Marketing Intelligence and Planning, Vol. 19, No. 4, pp. 254-262.

12 Dommeyer, C. J. and Moriarty, E. (2000) 'Comparing two forms of an e-mail survey: Embedded vs. attached', Journal of the Market Research Society, Vol. 42, No. 1, pp. 39-50.

13 Mehta, R. and Sivades, E. (1995) 'Comparing response rates and response content in mail versus 
electronic mail surveys', Journal of the Market Research Society, Vol. 37, No. 4, pp. 429-440.

14 Sheehan, K. B. and McMillan, S. J. (1999)

'Response variation in e-mail surveys: An exploration', Journal of Advertising Research, Vol. 39, No.4, pp. 45.

15 Wilson and Laskey, ref. 8 above.

16 Faught, K. S., Green, K. W. and Whitten, D. (2004) 'Doing survey research on the Internet: Yes, timing does matter', Journal of Computer Information Systems, Vol. 44, No. 3, pp. 26-34.

17 Wilson and Laskey, ref. 8 above.

18 Bachman, D. P., Elfrink, J. and Vazzana G. (2000) 'E-mail and snail mail face off in rematch', Marketing Research, Vol. 11, No. 3, pp. 11-15.

19 Kent, R. and Brandal, H. (2003) 'Improving e-mail response in a permission marketing context', International Journal of Market Research, Vol. 45, No. 4, pp. 489-496.

20 Roy and Berger, ref. 10 above.

21 Heerwegh, D., Vanhove, T., Matthijs, K. and Loosveldt, G. (2005) 'The effect of personalisation on response rates and data quality in web surveys', International Journal of Social Research Methodology, Vol. 8, No. 2, pp. 85-99.

22 Porter, S. R. and Whitcomb, M. E. (2003), 'The impact of contact type on web survey response rates', Public Opinion Quarterly, Vol. 67, No. 4, pp. 579-588.

23 Heerwegh et al., ref. 21 above

24 Heerwegh, D. (2005) 'Effects of personal salutations in e-mail invitations to participate in a web survey', Public Opinion Quarterly, Vol. 69, No. 4, pp. 588-598.

25 Dibb et al., ref. 2 above.

26 Mehta, R. and Sivades, ref. 13 above.

27 Kent, R. and Brandal, ref. 19 above.

28 Ibid.

29 Dommeyer and Moriarty, ref. 12 above.

30 Sheenah, K. B and Hoy, M. G. (2000) 'Dimensions of privacy concern among online consumers', Journal of Public Policy and Marketing, Vol. 19, No. 1, pp. 62-73.

31 Dibb et al., ref. 2 above.

32 Kent and Brandal, ref. 19 above.

33 Heberlein, T. A. and Baumgartner, R. (1978)

'Factors affecting response rates to mailed questionnaires: A quantitative analysis of the published literature', American Sociological Review, Vol. 43, No. 4, pp. 447-461.

34 Yammarino, F. J., Skinner, S. J. and Childers, T. L. (1991) 'Understanding mail survey response behavior', Public Opinion Quarterly, Vol. 55, No. 4, pp. 613-639.

35 Deutskens, E., De Ruyter K., Wetzels, M. and Oosterveld, P. (2004) 'Response rate and response quality of internet-based surveys: An experimental study', Marketing Letters, Vol. 15, No. 1, pp. 21-36.

36 Dommeyer and Moriarty, ref. 12 above.

37 Wilson and Laskey, ref. 8 above.

38 Deutskens et al. ref. 35 above.

39 Kanuk, L. and Berenson, C. (1975) 'Mail survey and response rates: A literature review', Journal of Marketing Research, Vol. 12, No. 4, pp. 440-453.

40 Fox, R. J., Crask, M. R. and Kim, J. (1988) 'Mail survey response rates', Public Opinion Quarterly, Vol. 52, No. 4, pp. 467-491.

41 Wilson and Laskey, ref. 8 above.

42 Schillewaert, N., Langerak, F. and Duhamel, T. (1998) 'Non-probability sampling for WWW surveys: A comparison', Journal of the Market Research Society, Vol. 40, No. 4, pp. 307-322.

43 Couper, M. P., Traugott, M. W. and Lamias M. J. (2001) 'Web survey design and administration', Public Opinion Quarterly, Vol. 65, No. 2, pp. 230-253.

44 Dibb et al., ref. 2 above.

45 Schillewaert et al., ref. 42 above.

46 Ibid.

47 Dibb et al., ref. 2 above.

48 Schillewaert et al., ref. 42 above.

49 Jackson, A. and DeCormier, R. (1999) 'E-mail survey response rates: Targeting increases response', Marketing Intelligence and Planning, Vol. 17, No. 3, pp. 135-139.

50 Wilson and Laskey, ref. 8 above.

51 Deutskens et al. ref. 35 above.

52 Yammarino et al., ref. 34 above.

53 Wilson and Laskey, ref. 8 above.

54 Dibb et al., ref. 2 above.

55 Kent and Brandal, ref. 19 above.

56 Heerwegh, ref. 24 above.

57 Ibid.

58 Cho, H. and LaRose, R. (1999) 'Privacy issues in Internet surveys', Social Science Computer Review, Vol. 17, No. 4, pp. 421-434.

59 Sheenah and Hoy, ref. 30 above. 